\title{
11 Investment incentives in auctions: an experiment
}

\author{
VERONIKA GRIMM, FRIEDERIKE MENGEL, \\ GIOVANNI PONTI AND LARI ARTHUR \\ VIIANTO*
}

We experimentally analyze first- and second-price procurement auctions where one bidder can achieve a comparative cost advantage by investment prior to the auction. Theory predicts that bidders invest more often prior to second-price auctions than prior to first-price auctions, which is clearly confirmed by our experimental data. Bidding in the auction (after investment) is more aggressive than the equilibrium prediction in both auction formats.

\section{Introduction}

Different market institutions provide different incentives for firms to engage in activities that affect their competitive positions. For example, prior to a procurement auction investments can be made either to reduce a firm's own cost of production, or even to raise the cost of possible competitors. Empirical evidence indicates that companies make use of this possibility extensively. ${ }^{1}$ Thus, both, auction rules and investment incentives have to be accounted for when it comes to compare revenue and efficiency of selling (or buying) institutions.

A number of papers theoretically analyze investment incentives in procurement auctions. Most of them assume that investment decisions are not observable prior to a competition. ${ }^{2}$ Then, a typical finding is that

* We thank Aitor Calo Blanco, Thyge Benned Jensen, Jaromir Kovárik and Ricardo Martinez for valuable research assistance. We also wish to thank seminar participants in Alicante, Istanbul (SCW 2006), Jena, Sevilla (SMYE 2006) and Valencia (EARIE 2007) for helpful comments. Financial Support by the Deutsche Forschungsgemeinschaft and the Instituto Valenciano de Investigaciones Económicas (IVIE) is gratefully acknowledged.

${ }^{1}$ De Silva, Dunne and Kosmopolou (2003).

2 See Tan (1992), Piccone and Tan (1996), and Bag (1997). 
investment is symmetric so that revenue equivalence between market institutions is preserved also in a model that allows for investment. This is not necessarily true if investment is observable. If firms strategically react to the decisions made at the investment stage, it is not immediately clear whether ex ante symmetry implies symmetric investment. For the case that only one firm has the possibility to invest, first- and second-price auctions with observable investment have been analyzed by Arozamena and Cantillon (2004). They show that equilibrium investment is lower prior to first-price auctions than prior to second-price auctions. The reason is that, in a first-price auction, a bidder's investment in his own comparative advantage makes his opponents' bids more aggressive. This strategic effect generally diminishes benefits from investment; it can even make investment undesirable.

In this chapter we experimentally investigate investment behavior prior to first- and second-price auction markets in a framework that is inspired by the model of Arozamena and Cantillon. Throughout the experiment we ran procurement auctions with two subjects each. Subjects played a two-stage game. At the first stage, one subject could invest in order to obtain a superior distribution of cost (not investing led to symmetry). At stage two, subjects competed in a procurement auction that was first or second price, depending on the treatment.

As expected, we find that bidding behavior at the auction stage does not perfectly coincide with equilibrium bidding. In the first-price auction, bidders bid more aggressively than predicted by the riskneutral Nash equilibrium. In the second-price auction, about half of the bids are close to equilibrium, while a rather large fraction of bids is below cost. Both observations are standard in the experimental literature on auctions. ${ }^{3}$ We find, however, that the relative investment incentives in the two auction formats are preserved given the observed behavior at the auction stage. That is, investment incentives given the actual play are still higher in the second-price than in the first-price auction, although investment is less profitable than predicted in both formats. At the investment stage we observe that, in accordance with the theory, investment is indeed lower prior to first-price auctions than prior to second-price auctions. Given the actual play in the auctions, investment is too high in both formats.

${ }^{3}$ For surveys, see Kagel (1995) and the chapter by Offerman and Onderstal in this volume. 
To summarize, our experimental evidence clearly shows that participants are aware of the strategic links between the investment and the auction stage. However, observed behavior does not perfectly coincide with the theoretical benchmark. We run two additional treatments to study the potential influence of the (existence of an) investment stage on bidding behavior in the auction more deeply. In our first control treatment we run asymmetric auctions with two players, where the strong player's cost advantage was exogenous. In our second control treatment the opponent's behavior was simulated by a computer algorithm programmed to play the (risk-neutral) symmetric Bayes-Nash equilibrium strategy in both stages. With few exceptions we find no significantly different investment or bidding behavior in either of the two control treatments.

Our chapter is, to the best of our knowledge, the first experimental study on the impact of investment on bidding behavior in procurement auctions. We chose procurement auctions (instead of auctions) since investment in cost reduction is a natural choice to present to participants. There are very few experimental studies of procurement auctions, and thus, our study (as a byproduct) demonstrates that behavior largely does not depend on the frame. There is, of course, a vast experimental literature on auctions, to which our chapter relates. ${ }^{4,5}$ The experimental study which is closest to ours is Güth et al. (2005), who study asymmetric first- and second-price auctions (without an investment stage) and afterwards elicit the participant's preferences for the auction format (first- or second-price auction). In this respect, their experimental environment corresponds to our treatment with exogenous investment (with the difference that they deal with a standard auction format) and our findings in this treatment confirm theirs.

The remainder of this chapter is organized as follows. In Section 2 we introduce the theoretical model that was the basis for our experimental design. The experimental design is presented in Section 3, and our hypotheses in Section 4. We report the results in Section 5 and conclude in Section 6. Experimental Instructions and Regression Tables can be found in the Appendix.

\footnotetext{
4 Kagel (1995).

5 In this respect, our evidence mostly confirms results from the experimental literature on "standard" auctions (take, for example, more aggressive bidding on behalf of the weak firm).
} 


\section{Investment incentives in procurement auctions}

\subsection{The game}

We consider a two-player, two-stage game. At stage one, one of two firms (firm 1) can affect its relative competitive position through an investment in cost reduction. At stage two both firms ( 1 and 2 ) compete in a procurement auction. Production costs are privately observed by the firms prior to the procurement auction at stage two. In the following we analyze behavior at both stages for two alternative auction formats, a second-price auction (SPA) and a first-price auction (FPA).

\section{Investment stage}

At the investment stage, one of the two firms, say firm 1, can make a decision that affects its production cost at stage two in a probabilistic sense. Investment is a binary choice to realize or not to realize a certain pre-specified reduction of (stage two) production cost. Investment is costly. Investment cost $k$ is observed only by firm 1 prior to its investment decision. Under these assumptions, firm 1's decision can be formalized by an investment function $\delta^{f}(k)$, where $\delta^{f}(k)=1$ $\left(\delta^{f}((k)=0)\right.$ denotes the decision (not) to invest when the investment cost is $k$ and the stage two auction format is $f \in\{F P A, S P A\}$. If $\delta^{f}(k)=1$ (i.e. firm 1 decides to invest), its production cost at stage two, $C_{1}$, is uniformly distributed in $[\underline{c}-w, \bar{c}]$. If $\delta^{f}\left((k)=0, C_{1}\right.$ is uniformly distributed in $[\underline{c}, \bar{c}]$. In either case, firm 2's production cost, $C_{2}$, is drawn uniformly from $[\underline{c}, \bar{c}]$. In other words, if firm 1 decides not to invest, firms are symmetric at stage two. In contrast, if firm 1 invests, its production cost is lower in the sense of first-order stochastic dominance and firms are asymmetric at stage two. ${ }^{6}$ In this latter case, we refer to firm $1(2)$ as the strong (weak) bidder.

\section{Auction stage}

Prior to bidding in the auction, costs $c_{1}$ and $c_{2}$ are realized according to the relevant distribution. ${ }^{7}$ Each firm observes its own production cost,

${ }^{6}$ Note that as we are dealing with procurement auctions, the distribution that is first-order stochastically dominated is advantageous.

7 We denote random variables by capitals and realizations by the corresponding lower case letters. 
but not the other firm's cost. We consider two auction formats, a firstprice auction (FPA), and a second-price auction (SPA).

\subsection{Bidding behavior, investment decisions and equilibrium payoffs}

We solve the model by backward induction, starting from stage two. We use the same parameters as in our experimental design, namely $\underline{c}=300, \bar{c}=400$ and $w=100 .^{8}$

\section{Stage two: Equilibrium bid functions}

As for SPA, bidding the observed production cost is a weakly dominant strategy, independently of whether firm 1 has invested or not. Thus, we have

$$
b_{i}^{S P A}\left(c_{i}\right)=c_{i}, i=1,2,
$$

where $b_{i}^{f}\left(c_{i}\right)$ is firm i's bid function under auction format $f$, conditional on her privately observed production $\cos t c_{i}$.

As for FPA, equilibrium bid functions differ in the symmetric and the asymmetric case. We focus on the risk-neutral symmetric BayesNash equilibrium. In the symmetric case (no investment by firm 1) equilibrium bid functions, for both firms, are

$$
b_{i}^{S P A}\left(c_{i}\right)=\frac{\left(\bar{c}+c_{i}\right)}{2}, i=1,2
$$

In the asymmetric case, equilibrium bid-functions are given by

$$
\begin{aligned}
& b_{1}^{F P A}\left(c_{1}\right)=\frac{200\left(6 c_{1}-2600+\sqrt{520000-2400 c_{i}+3 c_{1}^{2}}\right)}{3\left(c_{1}-400\right)} \\
& b_{2}^{F P A}\left(c_{2}\right)=\frac{200\left(6 c_{1}-2200+\sqrt{2400+3 c_{2}^{2}-44000}\right)}{3\left(c_{1}-400\right)}
\end{aligned}
$$

${ }^{8}$ While we could solve the model also for general values of our parameters, we believe that there is little value added by doing so. Using the parameter configuration of the experiment moreover facilitates interpretation of the data. All our results qualitatively also hold for different parameterizations. 


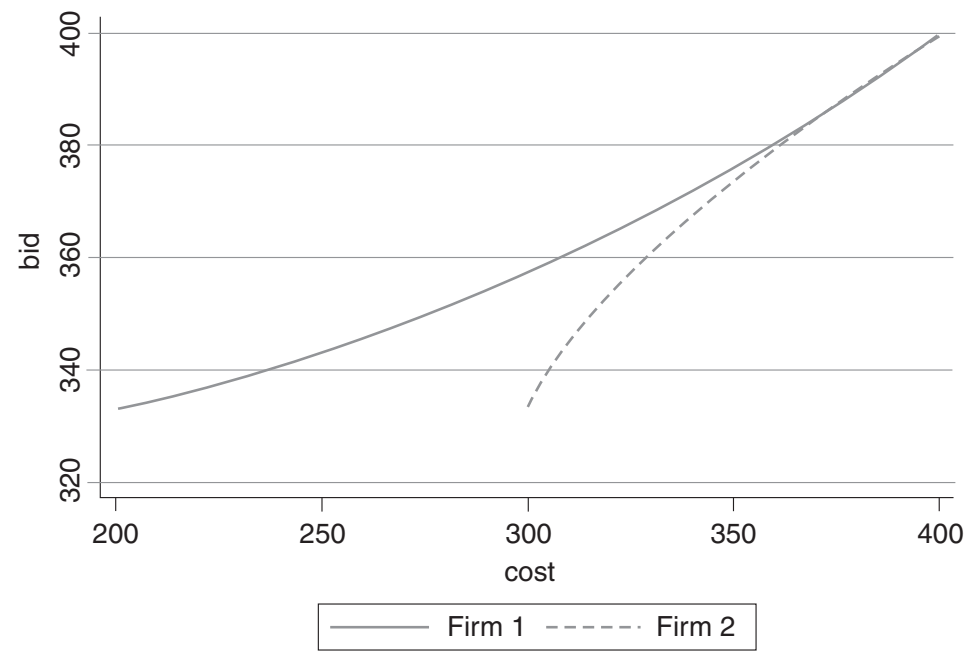

Figure 11.1 Equilibrium bid functions in the asymmetric FPA

Here $b_{1}^{F P A}\left(c_{1}\right)$ is the bid-function of a firm that has invested and thus, has the more advantageous cost distribution. See Figure 11.1.

Comparison of (3) and (4) yields that the advantaged bidder should bid point-wisely higher than the disadvantaged bidder, i.e. be less aggressive (recall that we consider a procurement auction). This implies that the ex ante disadvantaged bidder may sometimes win the auction although he observed a higher cost than his opponent. Consequently, the asymmetric first-price auction is inefficient with positive probability.

\section{Stage one: Optimal investment decision}

Given the above equilibrium bid functions at stage two, we can calculate the optimal investment decision at stage one. Whether it is beneficial for a firm to invest or not depends on how the firm's competitor reacts. Consequently in order to judge the profitability of an investment, one has to compute the equilibria at stage two for both the asymmetric case (corresponding to $\delta^{f}(k)=1$ ) and the symmetric case (corresponding to $\left.\delta^{f}(k)=0\right)$. Investment is profitable whenever investment cost is smaller than the difference between the expected profits obtained in the case of investing $(\delta=1)$ and in the case of not investing $(\delta=10)$. Let $\hat{k}_{f}$ denote the (equilibrium) investment cost threshold, under auction format $f \in\{F P A, S P A\}$, below which firm 1 should invest. 
Optimal investment decision rules, for the two auction formats, are given by

$$
\begin{aligned}
& \delta^{S P A}(k)=\left\{\begin{array}{cl}
1 & \text { if } \quad k<\hat{k}_{S P A}=125 / 3 \\
\{0,1\} & \text { if } k=\hat{k}_{S P A} \\
0 & \text { if } k>\hat{k}_{S P A} .
\end{array}\right. \\
& \delta^{F P A}(k)=\left\{\begin{array}{cl}
1 & \text { if } \quad k<\hat{k}_{F P A}=34 \\
\{0,1\} & \text { if } k=\hat{k}_{F P A} \\
0 & \text { if } k>\hat{k}_{F P A} .
\end{array}\right.
\end{aligned}
$$

Note that, since $\hat{k}_{F P A}=34<\hat{k}_{S P A}=125 / 3 \approx 41.67$, in FPA investment is profitable for a smaller range of parameters than in SPA. The reason for this difference is that in the first-price auction investment has a negative strategic effect (through a change in the opponent's bidding behavior), which is not the case in the second-price auction. This can be seen from equations (1) to (4). Investment has no effect on the competitor's bidding behavior in SPA, whereas in FPA it renders the competitor more aggressive in Nash-equilibrium. The reason is that firm 2 (the firm with the "worse" cost distribution) expects tougher competition in the auction than firm 1. Thus, in FPA investment has a drawback, since it makes the opponent relatively more aggressive.

\section{Bidders' equilibrium payoffs}

Let us finally present the bidders' equilibrium payoffs in our two auction formats. Payoff here means overall payoff of the entire game, including the investment stage. Obviously this payoff will depend on the cost threshold $\hat{k}_{f}$, as well as the cost of investment $k$ and the format of the auction. In Table 11.1 we summarize the firms' expected equilibrium payoffs in the first-price and in the second-price auction.

Note that in the symmetric case, both formats yield the same expected payoffs. ${ }^{9}$ In the second-price auction, investment by firm 1 decreases firm 2's payoff considerably, whereas in the first-price auction, bidder 2 even benefits from firm 1's investment. This is due to the negative strategic effect of investment.

\footnotetext{
9 This is due to the revenue equivalence theorem, see e. g. Myerson (1981).
} 
Table 11.1. Equilibrium payoffs

\begin{tabular}{llllll}
\hline & \multicolumn{2}{c}{ FPA } & & \multicolumn{2}{c}{ SPA } \\
\cline { 2 - 3 } \cline { 5 - 6 } & firm 1 & firm 2 & & firm 1 & firm 2 \\
\hline$\delta^{\mathrm{f}}(k)=1$ & $50.6-\mathrm{k}$ & 19.4 & & $58.3-\mathrm{k}$ & 8.3 \\
$\delta^{\mathrm{f}}(k)=0$ & 16.6 & 16.6 & & 16.6 & 16.6 \\
\hline
\end{tabular}

\section{The experimental design}

The experiment was conducted in seven sessions in May, 2005. A total of 168 students (24 per session) were recruited among the student population of the University of Alicante - mainly undergraduate students from the Economics Department with no (or very little) prior exposure to game theory. All monetary payoffs in the experiment were expressed in Spanish pesetas (€1 is approximately 166 ptas.). ${ }^{10}$ Average earnings were about $€ 18.5$, for an approximately 90 -minute experiment.

Table 11.2 shows our six different treatments. FPA and SPA are the initial treatments with endogenous investment. In treatments EXFPA and EXSPA the asymmetries that could be induced by investment in the first two treatments were exogenously given. In COMPFPA and COMPSPA the opponent of each player was played by a computerized agent. $^{11}$

We ran two sessions each of FPA and SPA and one session each of EXFPA and EXSPA, generating six and three independent observations per treatment, respectively (compare Table 11.2). As for COMPFPA and COMPSPA we ran one session only where twelve participants played each auction format, yielding twelve independent observations.

${ }^{10}$ It is standard practice, for all experiments run in Alicante, to use Spanish ptas. as experimental currency. The reason for this design choice is twofold. First, it mitigates integer problems, compared with other currencies (USD or Euros, for example). On the other hand, although Spanish pesetas are no longer in use (substituted by the Euro in the year 2001), Spanish people still use Pesetas to express monetary values in their everyday life. In this respect, by using a "real" (as a opposed to an artificial) currency, we avoid the problem of framing the incentive structure of the experiment using a scale (e.g. "Experimental Currency") with no cognitive content.

11 In the treatment with computerized opponents participants were informed that the computer is preprogrammed to play an optimal strategy. 
Table 11.2. The experimental treatments

\begin{tabular}{lll}
\hline & First-price auction & Second-price auction \\
\hline Stage 1: Investment & FPA & SPA \\
Stage 2: Auction & (6 indep. observations) & (6 indep. observations) \\
Exogenous advantage & EXFPA & EXSPA \\
Asymmetric auction & (3 indep. observations) & (3 indep. observations) \\
$\begin{array}{l}\text { Stage 1: Investment } \\
\text { Stage 2: Auction against }\end{array}$ & COMPFPA & COMPSPA \\
computerized agent & (12 indep. observations) & (12 indep. observations) \\
\hline
\end{tabular}

The seven experimental sessions were computerized. ${ }^{12}$ Written instructions were distributed at the beginning of the experiment and read aloud. At the end of the instructional phase, participants had to answer a set of control questions, to make sure that they had properly understood the key features of the experiment. ${ }^{13}$

In each session, participants played one of the six treatments for a total forty-four rounds. In the first six sessions, participants were divided into three cohorts of eight. Participants from different cohorts never interacted with each other throughout the experiment. In each round, participants from a cohort were randomly matched in pairs of two, where each subject played as firm 1 (2) every other round. This was done to enhance the subjects' understanding of strategic effects of investment decisions. Let time interval $\tau_{m}$ be the m-th block of eleven rounds such that $\tau_{1}$ refers to rounds $1-11, \tau_{2}$ refers to rounds 12 to 22 , and so on. Within each time interval $\tau_{m}$, participants acting as firm 1 experienced each and every possible investment cost of $k \in\{0,5, \ldots$, $45,50\}$ ptas. The sequence of costs was randomly selected within each time interval and was different for each cohort and period. After being told the current investment cost $k$, firm 1 had to decide whether to invest. By this design, we are able to characterize four complete investment functions $\delta^{f}(k)$, one for each time interval.

12 The experiment was programmed and conducted with the software z-Tree (Fischbacher, 2007).

13 The instructions for FPA, translated into English, can be found in the Appendix. Instructions for the remaining treatments are available upon request. 
Participants participating in the experiment initially received 1,000 ptas. These stakes were chosen to exclude the possibility of bankruptcy. Within each round, each subject received an additional fixed endowment of 10 ptas., while only those who had the opportunity to invest additionally received a fixed payment equivalent to the investment cost $k$ that they had in the current round. ${ }^{14}$

After each of the forty-four rounds, participants were informed of the identity of the stage two auction winner and her own monetary payoffs, as well as all the accumulated monetary payoff so far. The same information was also given in the form of a history table, so that participants could easily review the results of all the rounds that had been played so far.

\section{Hypotheses and research questions}

The theoretical analysis of Section 2 yields the following working hypotheses for our experiment.

(H1) Bidding behavior. Irrespective of the treatments we expect the following bidding behavior at stage two:

(i) In FPA we expect bidding behavior of strong bidders to be less aggressive than that of weak bidders in the sense that, for any given level of the production cost $c$, the strong bidder's bid should be higher.

(ii) In SPA, both strong and weak firms should always bid their production cost $c$.

(H2) Payoffs.

(i) In treatments FPA, SPA, COMPFPA and COMPSPA, if firm 1 does not invest, firms' payoffs should be the same. This is a consequence of the revenue equivalence theorem, since, in this case, bidders are symmetric. ${ }^{15}$

(ii) In all treatments, if firm 1 invests, her profits should be higher than firm 2's, and also higher than in the symmetric case.

14 The role of this addition nal payment is to ensure that a bidder who had invested in FPA (SPA) had the same wealth as a bidder who received the advantage for free in EXFPA (EXSPA). Also the risk of bankruptcy is reduced.

15 This argument does not apply for EXFPA (EXSPA) where, by construction, all auctions are asymmetric. Also note that revenue equivalence can only be expected conditional on the fact that no investment has taken place. 
Compared to the symmetric case, firm 2's profits in the asymmetric case are lower (higher) in SPA (FPA). From the auctioneer's point of view, FPA yields a lower procurement cost than SPA (for our particular parametrization). ${ }^{16}$

(H3) Investment behavior. Investment incentives are higher in treatments SPA and COMPSPA than in treatments FPA, COMPFPA. This is because, by (5)-(6), firm 1's optimal "threshold" strategy anticipates that, in FPA, firm 2 will bid more aggressively in reaction to firm 1's investment (something that does not happen in SPA).

(H4) Efficiency. By (1), the SPA outcome is efficient, independently of whether bidders are asymmetric or not. By contrast, in FPA, the allocation is predicted to be efficient only if firm 1 has not invested (i.e. when bidders face a symmetric auction).

In addition, by comparing treatments FPA and SPA to our four control treatments, we will try to answer the following (more behaviorally oriented) questions:

(HB1) Does the origin of the comparative advantage matter? That is, does the existence of the investment stage per se affect behavior at the auction stage?

(HB2) How does the strategic uncertainty affect participants'behavior? That is, do participants behave differently in treatments FPA (SPA) and COMPFPA (COMPSPA) because of strategic uncertainty?

\section{Experimental results}

In this section we report our experimental results. We first test our main hypotheses, $\mathrm{H} 1$ to $\mathrm{H} 4$, based on the evidence from treatments FPA and SPA in Sections 5.1 to 5.4.Then, in Section 5.5, we report evidence from the remaining four treatments to discuss whether and how the existence of an investment stage affects behavior, apart from what is theoretically predicted.

16 In general, revenues do not compare in an unambiguous way in asymmetric auctions. See Maskin and Riley (2000). 


\subsection{Bidding behavior (H1)}

Bidding behavior in the symmetric auctions (no investment) resembles very much what has been found in the experimental literature so far. In what follows we concentrate on the bidding behavior if the bidders have invested.

\section{Fpa}

Figures 11.2, 11.3 and 11.4 describe the participants' bidding behavior in FPA. We take three snapshots of the data: in Figure 11.2 (3) we plot a scatter diagram of player 1's (2's) bidding behavior after investment took place in stage one. In Figure 11.4 we report participants' bidding behavior (irrespective of their player position) if investment did not take place.

Figures 11.2 to 11.4 share the same structure. Every point of the scatter diagram corresponds to a cost-bid pair (i.e., an individual observation). The two continuous lines correspond to (i) the equilibrium bid function derived in Section 2 and (ii) production cost $c$ (which corresponds to the lower bound for any rationalizable bid).

As Figures 11.2 to 11.4 show, participants generally bid above cost, but below the equilibrium prediction, which is in line with most



Figure 11.2 Bidding behavior in FPA, firm 1 


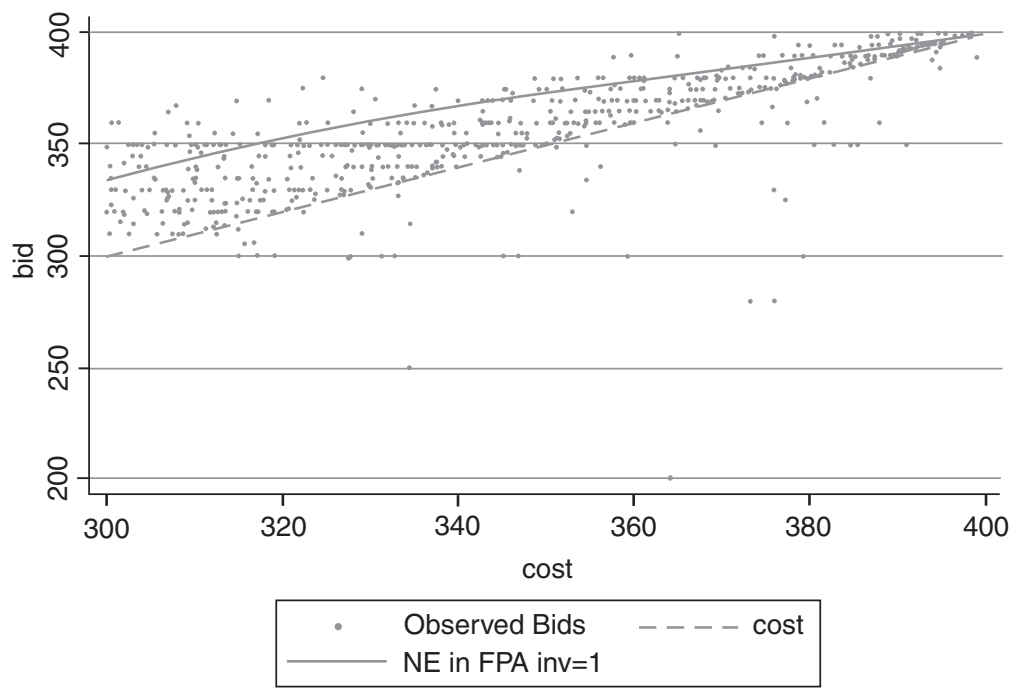

Figure 11.3 Bidding behavior in FPA, firm 2

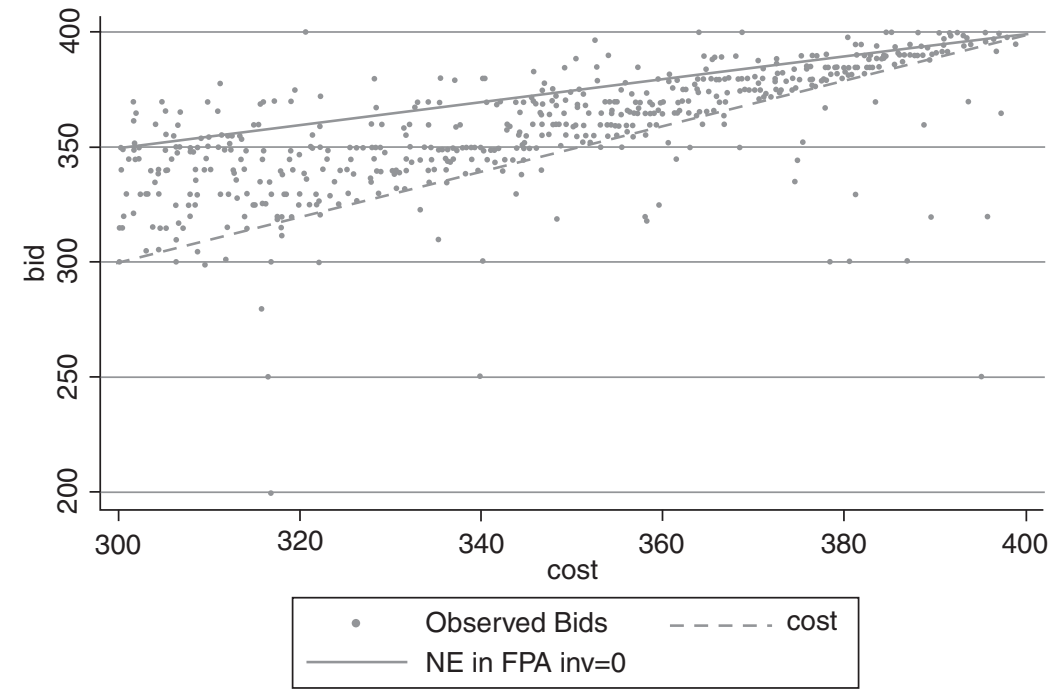

Figure 11.4 Bidding behavior in FPA, symmetric case 
experimental findings. ${ }^{17}$ After investing at stage one, firm 1 should be aware of the fact that firm 2's cost can never fall below 300. As a matter of fact, as Figure 11.2 shows, $b_{1}=300$ seems to be a "focal bid" for cost realizations below $300 .^{18}$ Over time bidders seem to learn to bid somewhat closer to equilibrium, although this (learning) effect is not significant.

To estimate participants' aggregate bid functions, we employ, for all treatments, the same simple random effects linear regression. The underlying model assumes that participants use bid functions that are two-piecewise linear, with a structural break fixed at $c=300$ :

$$
\begin{aligned}
b_{i t}= & a_{0}+\beta_{0} c_{i t}+a_{1} \delta_{1}+\beta_{1} \delta_{1} c_{i t}+a_{2} \delta_{1} \delta_{c+}+\beta_{2} \delta_{1} \delta_{c+} c_{i t} \\
& +\varepsilon_{i}+\varepsilon_{i t}
\end{aligned}
$$

where $\delta_{1}=1\left(\delta_{1}=0\right)$ if firm 1 has (not) invested at stage one, $\delta_{c+}=1$ $\left(\delta_{c+}=0\right)$ if $c \geq(<) 300, \varepsilon_{i}$ is the individual (random) component which describes subject $i$ 's unobserved time-invariant heterogeneity and $\varepsilon_{i t}$ is an idiosyncratic error term.

Table 11A.1 (in the appendix) reports the estimation results for regression (7) in the case of treatments FPA and SPA. ${ }^{19,20}$ Comparing the estimated bid functions with our theoretical prediction we notice that our estimated bid functions are always (point-wisely) significantly different from their corresponding equilibrium levels. In particular, for both, firm 1 and firm 2, bidding behavior is always more aggressive than predicted, as the estimated bid functions are always (pointwisely) significantly below equilibrium. Moreover (which is consistent with theory), firm 2 behaves more aggressively than firm 1 in the

17 See, for example, Kagel (1995), or, for asymmetric auctions, Güth et al. (2005).

18 This consideration notwithstanding, we also see a significant proportion of bids below 300 (6.2\% of total observations), although these observations mainly correspond to the first periods (i.e. in time interval 4 , only $2.7 \%$ of total observations were bids below 300).

19 We also included time dummies for our four time intervals $\tau_{m}, m=1, \ldots, 4$. The corresponding coefficients (also for interaction terms) turned out not to be significant. I.e., we observe no particular time trends in either direction.

20 The estimation of the variance-covariance matrix of (7) has been adjusted to control for possible correlation among observations drawn from the same matching group. In other words, our estimations are performed under the assumption that the history of each matching group (and not the history of the eight participants that form each matching group) corresponds to an independent observation. 


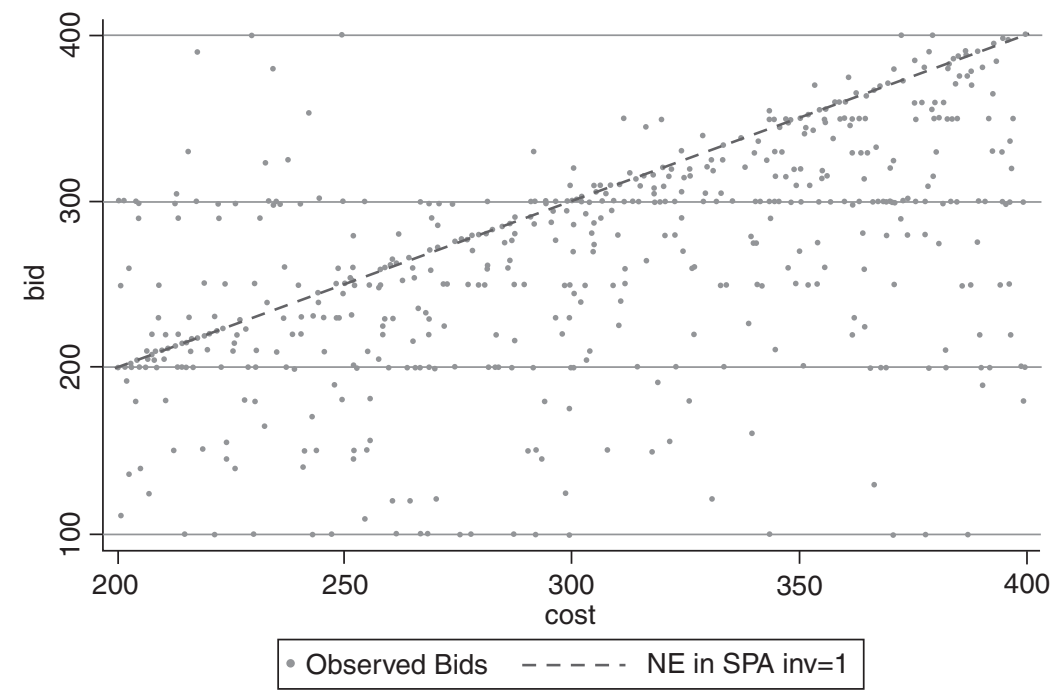

Figure 11.5 Bidding behavior in SPA, firm 1

asymmetric auction. As for firm 1, we observe that bidding behavior is somewhat "closer" to equilibrium when $c<300$, i.e. the difference between theoretical and estimated bids for $c<300$ is much smaller than the corresponding difference for $c \geq 300$ (30.9 versus 42.88). This evidence seems to suggest that, whenever investment yields a dominant position for firm 1 (i.e. production cost $c<300$ ), firm 1's bidding is comparatively less aggressive than when investment does not yield such a clear cooperative cost advantage.

\section{Spa}

Figures 11.5, 11.6 and 11.7 report the same information as Figures 11.2 to 11.4 for the SPA sessions. As Figures 11.5 to 11.7 show, bidding behavior is much noisier in SPA than in FPA, in particular (for both player positions) after firm 1 has invested in stage 1 . As a consequence, the fit of our linear regressions in Table 11A. 1 (overall $R^{2}=0.5427$ for firm 1 and 0.5127 for firm 2 in FPA, against 0.4294 and 0.1295 in the SPA case, respectively), drops dramatically. This translates into much higher standard errors in the estimated coefficients. This consideration notwithstanding, we see that, once again, participants bid below 


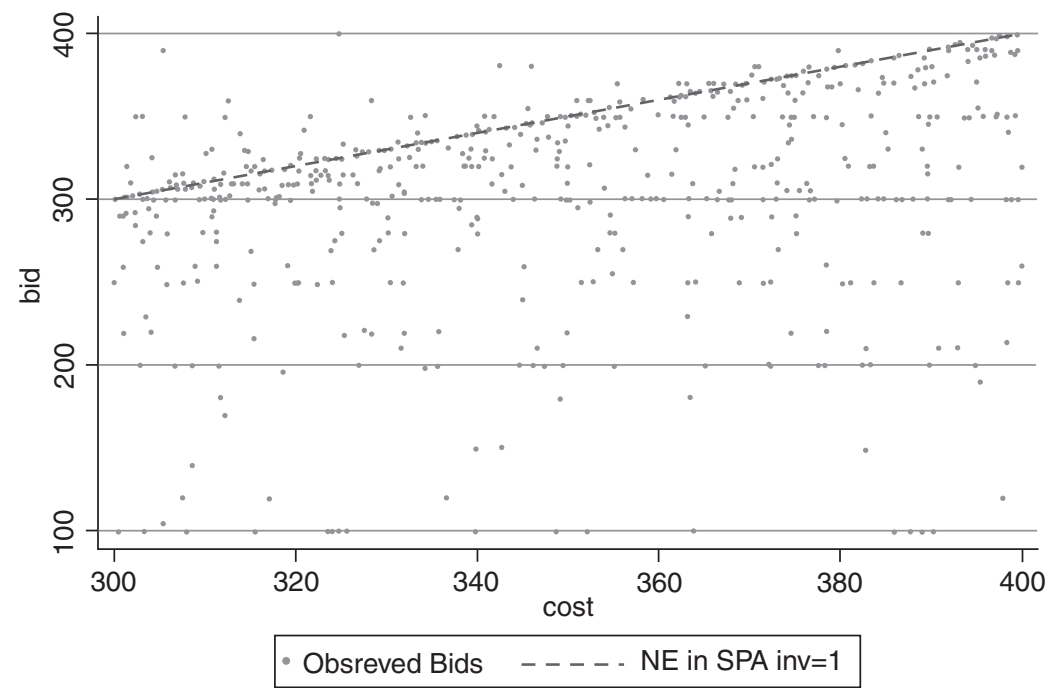

Figure 11.6 Bidding behavior in SPA, firm 2

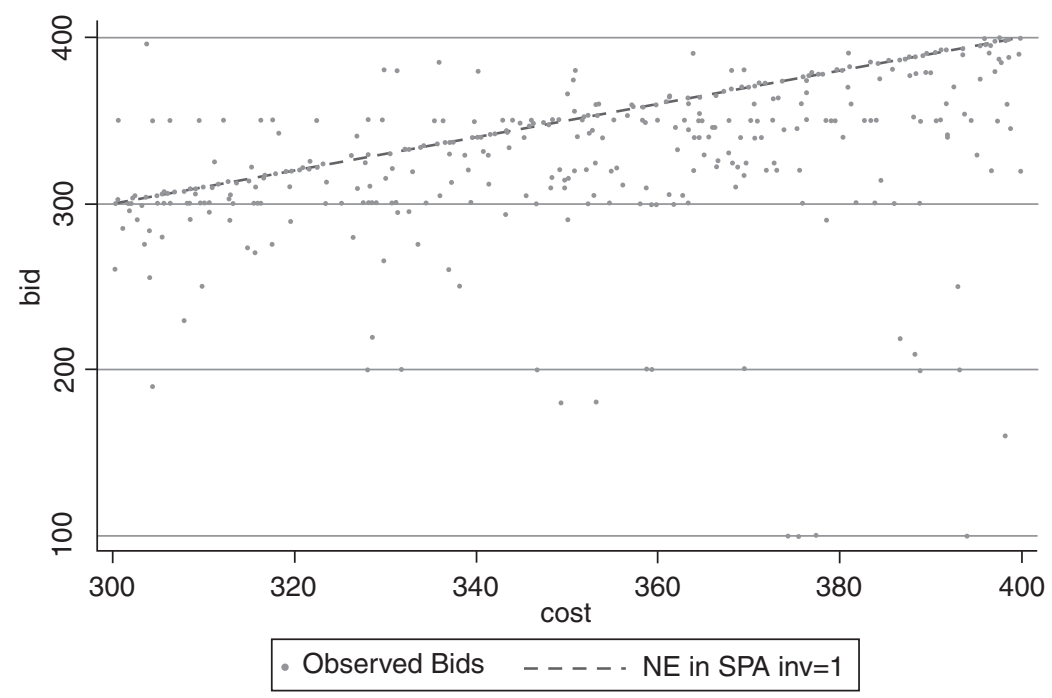

Figure 11.7 Bidding behavior in SPA, symmetric case 
Table 11.3. Proportions of equilibrium bids and over-/underbidding in SPA when $\delta=1$

\begin{tabular}{llll} 
& underbidding & equilibrium bidding & overbidding \\
\hline & $<$ cost $-1 \%$ & $=$ cost $+/-1 \%$ & $>$ cost $+1 \%$ \\
all periods & $49.76 \%$ & $41.34 \%$ & $8.90 \%$ \\
time interval 4 & $46.21 \%$ & $47.35 \%$ & $6.44 \%$ \\
\hline
\end{tabular}

equilibrium (i.e. they behave more aggressively than predicted), and this difference is (point-wisely) statistically significant. ${ }^{21}$

Such lower levels of $R^{2}$ "between participants", calls for further analysis of our evidence, looking at whether high variance in the data has to be attributed to heterogeneity in our subject pool, rather than heterogeneous responses (of each single individual participating in the experiment) to similar cost levels. As it turns out, participants were indeed quite heterogeneous. Roughly speaking, they can be classified in four types: (i) those who played equilibrium from the very beginning $(27 \%)$, (ii) those who learned how to play the equilibrium over time $(25 \%)$, (iii) those who consistently underbid throughout $(31 \%)$, and (iv) those who alternate over- and underbidding across time (17\%).

Table 11.3 summarizes the proportions of bids equal to observed cost $+/-1 \%$, above and below, respectively. As Table 11.3 shows, the percentage of bids close to the theoretical prediction mildly increased over time. Still, more than half of the bidders over- or underbid their cost even in time interval 4 , where the vast majority underbid their cost (i.e. run the risk to incur a loss). Over all time intervals, we observe $8.9 \%$ of bids below 200, and an additional $24.76 \%$ of bids between 200 and 300 that were more than $10 \%$ below the corresponding cost. A large proportion of the extremely low bids (below 200 ptas.) were made by three bidders only. In addition, the number of bids below 200 is much higher in the case where player 1 had invested. Then, there are $14.6 \%$ firm 1 bids and $6.4 \%$ firm 2 bids in this range. In the symmetric case, there are only $3 \%$ bids by firm 1 and $3.5 \%$ bids by firm 2 below 200 .

The observed behavior at the auction stage is in line with the experimental literature. For example, Güth et al. (2005) report that

21 This is in line with previous experimental evidence on the second-price auction. 
Table 11.4. Observed and equilibrium payoffs in FPA and SPA

\begin{tabular}{clccccc}
\hline & \multicolumn{2}{c}{ FPA } & & \multicolumn{2}{c}{ SPA } \\
\cline { 3 - 3 } \cline { 5 - 6 } & & firm 1 & firm 2 & & firm 1 & firm 2 \\
\hline \multirow{2}{*}{$\delta=1$} & observed & 29.097 & 2.261 & 36.007 & -3.160 \\
& (std. dev.) & $(41.673)$ & $(18.492)$ & & $(57.775)$ & $(32.256)$ \\
& equilibrium & 50.6 & 19.4 & & 58.3 & 8.3 \\
& observed & 5.053 & 6.196 & 6.693 & 6.465 \\
$\delta=0$ & (std. dev.) & $(18.337)$ & $(16.940)$ & & $(28.143)$ & $(25.844)$ \\
& equilibrium & 16.6 & 16.6 & & 16.6 & 16.6 \\
\hline
\end{tabular}

half of their participants bid approximately truthfully. Underbidding in their experiment was slightly less prominent. They argue that this is due to the fact that in their experiments participants had previously gained experience because they had to participate in several first-price auctions prior to playing SPA.

\section{Result 1 (bidding behavior)}

(i) In FPA bidding is more aggressive than the equilibrium prediction for all types.

(ii) In SPA bidding behavior is highly heterogeneous. While approximately half of the subjects bid their cost or learn to do so over time, $50 \%$ of subjects over- or underbid considerably, where underbidding is by far the most prominent pattern.

\subsection{Payoffs (H2)}

Table 11.4 contains information about average bidder payoffs in FPA and SPA from the auction at the second stage. Investment costs are not taken into account. (This is because investment costs are sunk at this stage and should not affect payoffs in the auction.)

In the symmetric case (i.e. when $\delta=0$ ), there are no significant differences between FPA and SPA, as predicted by the revenue equivalence theorem. In both the symmetric and the asymmetric auctions, however, participants bid much more aggressively than predicted, with the consequence that payoffs are lower than their theoretical values. 
Table 11.5. Procurement cost in FPA and SPA

\begin{tabular}{llll}
\hline & & FPA & SPA \\
\hline \multirow{4}{*}{$\delta=1$} & observed & 327.630 & 333.024 \\
& (std. dev.) & $(33.819)$ & $(44.620)$ \\
& equilibrium & 354.1 & 358.3 \\
& observed & 348.074 & 353.931 \\
$\delta=0$ & (std. dev.) & $(24.985)$ & $(32.177)$ \\
& equilibrium & 366.7 & 366.7 \\
\hline
\end{tabular}

In the asymmetric auctions (i.e. when $\delta=1$ ), firm 1's payoff is significantly higher in SPA than in FPA. While, as predicted, firm 2's payoff is significantly higher in FPA than in SPA, also for firm 2 actual payoffs are dramatically lower than their theoretical predictions. In SPA, firm 2's average payoff is even negative (which is due to the excessive underbidding of valuations reported in the previous section). In FPA, firm 2's payoff should theoretically be higher than either firm's payoff in the symmetric case. However, in our experiment the opposite is true. This is presumably due to the extremely aggressive behavior of weak bidders in the FPA format.

Note that comparison of the symmetric and the asymmetric payoffs determines whether investment at stage one is profitable or not. Investment is profitable whenever the investor's additional expected payoff in case of investment is higher than investment cost. The aggregate data (over all time intervals) suggest that investment incentives given the observed behavior are lower than their theoretical prediction. The critical investment cost suggested by aggregate data is 24.044 (theoretical prediction: 34) for FPA and 29.314 (theoretical prediction: 41.667) for SPA. Still, according to the observed payoff differences the relative profitability of investment (which theoretically is higher in the second-price auction) is preserved.

Table 11.5 finally reports the procurement cost in all four auction types together with their theoretical predictions. As expected, procurement cost in both formats is significantly lower if an investment has been made. Revenue equivalence (i.e. the hypothesis that procurement cost in SPA equals procurement cost in FPA) has to be rejected at the $5 \%$ level in either case, $\delta=1$ and $\delta=0$. 
Table 11.6. Predicted cost thresholds from a logit regression for both FPA and SPA

\begin{tabular}{llllll}
\hline & $\tau_{1}$ & $\tau_{2}$ & $\tau_{3}$ & 4 & prediction \\
\hline FPA & 46.71 & 39.42 & 34.93 & 35.88 & 34 \\
Threshold given observed bids & 15.18 & 22.78 & 31.41 & 26.18 & \\
SPA & 50 & 50 & 44.20 & 43.60 & 41.67 \\
Threshold given observed bids & 32.12 & 26.12 & 30.22 & 41.65 &
\end{tabular}

\section{Result 2 (payoffs)}

(i) Bidder payoffs are significantly lower than their theoretical predictions, both in the symmetric and the asymmetric auctions. In the symmetric auctions payoffs do not differ between FPA and SPA. In the asymmetric auctions bidder payoffs are higher in SPA than in FPA for firm 1 and vice versa for firm 2.

(ii) Procurement cost is lower in the asymmetric than in the symmetric auction and among the asymmetric auctions lower in FPA.

\subsection{Investment behavior (H3)}

As theory predicts, our subjects' investment behavior was influenced by both the investment cost $k$ as well as the auction format (FPA or SPA), where the probability of investment was significantly higher in SPA. This seems to suggest that players were able to estimate that the value of such an investment is higher on average in SPA than in FPA. In SPA the majority of subjects invested for almost any level of $k$ at the beginning of the experiment. That is, they invested "too much" both with respect to equilibrium behavior and expected returns, given bidding behavior at stage 2 . In later rounds they gradually reduced their propensity to invest, and aggregate behavior became close to the theoretical prediction.

Table 11.6 reports the predicted cost thresholds as predicted by a logit regression. Estimated thresholds reported in the table indicate the maximum cost level for which "not invest" corresponds to the modal decision.

As Table 11.6 shows, predicted cost thresholds decrease over time until they reach 35.88 in FPA and 43.60 in SPA. Whereas in the first 
twenty-two rounds of FPA and the first thirty-three rounds of SPA it is significantly higher than the threshold bidders should have had, given the actual bidding behavior at stage 2 (see Table 11.6), it is close to this threshold for all other rounds. The two threshold values (i.e. the actual threshold and the optimal threshold given average observed behavior) display a tendency to converge towards the end of the experiment.

\section{Result 3 (investment)}

Investment starts out at high levels and over time approaches the theoretical prediction as well as the threshold given the observed behavior.

\subsection{Efficiency (H4)}

According to the equilibrium prediction, all auctions but the asymmetric FPA should yield an efficient allocation. That is, the bidder with the lower cost should win the auction. Because costs were randomly and independently drawn in our experiment, simply comparing treatments with respect to the achieved production cost would be biased by these random draws. For this reason, we compare the auction formats with respect to three different efficiency measures that are aimed to minimize this bias:

- Allocative efficiency: the number of efficiently allocated units (i.e. to the bidder with the lower cost) relative to the total number of units.

- Relative efficiency loss: the loss in terms of total production cost relative to the maximum possible efficiency loss.

- Relative efficiency: the minimal possible production cost relative to the achieved total production cost.

Allocative efficiency does not reflect the actual magnitude of efficiency losses due to misallocations. If the "wrong" bidder obtains a unit, his cost may be substantially or only slightly above the other bidder's cost, raising production cost either dramatically or only slightly. Our second and third measures take this into account. In Table 11.7 we report for each measure aggregate results over all pairs and time intervals, as well as for time interval 4. 
Table 11.7. Efficiency, measured by allocative efficiency, relative efficiency loss and relative efficiency

\begin{tabular}{llccl}
\hline & Periods & $\begin{array}{l}\text { Allocative } \\
\text { efficiency }\end{array}$ & $\begin{array}{l}\text { Relative } \\
\text { efficiency loss }\end{array}$ & $\begin{array}{l}\text { Relative } \\
\text { efficiency }\end{array}$ \\
\hline FPA & all & $\mathbf{8 5 . 9 9 \%}$ & $\mathbf{8 . 4 6 \%}$ & $\mathbf{9 8 . 0 8 \%}$ \\
$\delta=1$ & $34-44\left(\tau_{4}\right)$ & $86.21 \%$ & $9.66 \%$ & $97.82 \%$ \\
SPA & all & $78.88 \%$ & $14.16 \%$ & $96.83 \%$ \\
$\delta=1$ & $34-44\left(\tau_{4}\right)$ & $86.17 \%$ & $6.65 \%$ & $98.40 \%$ \\
FPA & all & $83.62 \%$ & $10.04 \%$ & $98.98 \%$ \\
$\delta=0$ & $34-44\left(\tau_{4}\right)$ & $85.56 \%$ & $9.03 \%$ & $99.08 \%$ \\
SPA & all & $73.59 \%$ & $20.46 \%$ & $98.01 \%$ \\
$\delta=0$ & $34-44\left(\tau_{4}\right)$ & $73.33 \%$ & $21.66 \%$ & $97.97 \%$
\end{tabular}

We observe that allocative efficiency (the percentage of Paretoefficient allocations) is lower in the asymmetric second-price auction than in the first-price auction. However, the difference disappears if one considers only time interval 4. Thus, while the erratic behavior of many subjects in SPA seems to partly disappear over time, allocative efficiency stays constant in the asymmetric first-price auction, where it coincides with the equilibrium prediction. The effect appears more pronounced if we look at the relative efficiency loss. Here, the efficiency loss increases in FPA, while it decreases remarkably in the asymmetric second-price auction.

The remarkably low efficiency values especially for the symmetric SPA may be due to a small sample size together with a self-selection problem: since in SPA investment was almost always profitable, the group that played the symmetric SPA may have contained a large fraction of subjects that did not understand this. Overall, the efficiency rates are remarkably close to those found by Güth et al. (2005), again accounting for the fact that their SPA-players were more experienced than ours.

\section{Result 4 (efficiency)}

Efficiency is initially lower in the asymmetric SPA than in the asymmetric FPA. Over the course of the experiment, this result is reversed. 


\subsection{Robustness of the results}

Does the origin of the comparative advantage matter? (HB1)

To answer this question we ran two treatments (EXFPA and EXSPA) where bidders played an asymmetric auction without a preceding investment stage. That is, firm 1 had cost uniformly distributed in [200, 400], while firm 2's cost distribution was uniform in [300, 400]. This is exactly the situation subjects faced in our original treatments in case the investor actually decided to invest. Theoretically, thus, bidders should behave identically in EXFPA (EXSPA) and FPA (SPA). Our data largely confirm the theoretical hypothesis.

Table 11A.2 (in the Appendix) reports estimates of a two-piecewise linear bid function of firm 1 and 2, pooling (in case of investment on behalf of firm 1) observations from FPA (SPA) and EXFPA (EXSPA). We only find a significant (point-wise) difference in the estimated bid functions for firm 1 in SPA when $c$ is sufficiently high $(c>317$ approx.). In all other cases, subjects do not behave differently in either treatment protocol. In particular in a first-price auction, the existence of an investment stage does not per se affect behavior in the auction.

How does the strategic uncertainty affect subjects' behavior? (HB2)

We address the second question by comparing our results with those coming from treatments COMPFPA and COMPSPA, where subjects bid against computerized agents playing the RNNE strategy described in Section 2 at both stages.

Table 11A.3 (in the Appendix) reports estimates analogous to those of Table 11A.2 only for those observations for which investment took place. Again, we find no significant differences for the first-price auction, while in second-price auctions firm 1 bids significantly less aggressively (i.e. closer to the equilibrium prediction) in COMPSPA than in SPA for sufficiently high cost levels.

Recall, however, that bidders are rather heterogeneous in SPA and therefore, the bid function of a representative agent might not reflect the behavior of many players. A categorization in bidding types reveals that there are more "rational" players (i.e. types that play equilibrium from the beginning or learn to do so over time) in both control treatments (EXSPA and COMPSPA) than in SPA (see Table 11.8).

This also reflects in the rates of bidders who bid their cost, and over/ underbid, respectively (see Table 11.9). Obviously it is not the 
Table 11.8. Bidder types in SPA (EQ: subjects who bid their cost from the beginning, L: those who learned the equilibrium over time, $U$ : those who always underbid, U/O: those who alternated under and overbidding)

\begin{tabular}{lllll}
\hline & EQ & $\mathrm{L}$ & $\mathrm{U}$ & $\mathrm{U} / \mathrm{O}$ \\
\hline SPA & $27 \%$ & $25 \%$ & $31 \%$ & $17 \%$ \\
EXOSPA & $37.5 \%$ & $29.2 \%$ & $20.8 \%$ & $12.5 \%$ \\
COMPSPA & $41.6 \%$ & $25 \%$ & $25 \%$ & $8.3 \%$ \\
\hline
\end{tabular}

Table 11.9. Proportions of equilibrium bids and over-/underbidding for the three SPA treatments - all periods

\begin{tabular}{llll}
\hline treatment & underbidding & equilibrium bidding & overbidding \\
\hline SPA & $<\operatorname{cost}-1 \%$ & $=\operatorname{cost}+/-1 \%$ & $>\operatorname{cost}+1 \%$ \\
EXSPA & $49.76 \%$ & $41.34 \%$ & $8.90 \%$ \\
COMPSPA & $33.62 \%$ & $55.68 \%$ & $10.70 \%$ \\
& $42.23 \%$ & $51.51 \%$ & $6.25 \%$
\end{tabular}

strategic uncertainty that drives the difference, since in COMPSPA bidders do not bid truthfully more frequently than in EXSPA. A possible explanation could be that players learn the optimal strategy better whenever a) they play against more rational players (as is the case in COMSPA) and b) whenever they face a smaller strategy space and thus a smaller "cognitive load" (as is the case in EXSPA).

\section{Conclusion}

In this chapter we have experimentally investigated behavior in firstand second-price procurement auctions where one bidder had the possibility to improve his competitive position by investing. Our results are in line with several qualitative predictions of the theory. In particular, we observe that:

- Subjects invest more often prior to SPA than prior to FPA. Over time investment levels approach the theoretical prediction, as well as the optimal threshold given the observed data. 
- Procurement cost is lower in the asymmetric FPA than in the asymmetric SPA (and bidder payoffs are also lower in FPA).

- In FPA, weak bidders bid relatively more aggressively than strong bidders.

- In those cases where investment had taken place, efficiency is initially lower in SPA. This turns around in the course of the experiment such that finally efficiency is lower in FPA than in SPA, as predicted.

In line with the experimental literature on auctions, we find that in FPA, bidding is more aggressive than predicted, resulting in very low bidder payoffs and procurement cost. In SPA, bidders are highly heterogeneous with approximately half of them playing close to the equilibrium prediction, while the majority of the remaining subjects underbid. On average this also leads to lower bidder payoffs and procurement cost in SPA.

Our results yield several conclusions for public policy. Obviously, if investment prior to the auction is important or if positive synergies between auctions exist it is not immediately obvious whether first- or second-price auctions are better suited for public procurement. Firstprice auctions are typically easier understood by the subjects, yield low procurement cost (since bidders bid persistently more aggressively than predicted), and are less susceptible to collusion than second-price auctions. Second-price auctions, on the other hand, imply higher investment incentives. Thus, if improvement of production technologies or process innovations are important, second-price auctions are likely the better choice. Our analysis (as many other experiments) demonstrates, however, that the second-price auction format has to be carefully chosen, as typically a large share of subjects do not easily understand the equilibrium strategy.

\section{Appendix A: Optimal investment}

\section{Second-price auction}

To find the optimal investment decision rule in the second-price auction, we solve the model backwards. Note that the expected payoff of a player 1 is given by

$$
B\left(\delta^{S P A}\right)=\Pi_{1} E\left(C_{2}-C_{1} \mid C_{1}<C_{2}\right)
$$


where $E$ denotes the expectations operator and $\Pi_{1}$ denotes the probability for player 1 to win the auction. ${ }^{22}$ Obviously both $\Pi_{1}$ and will $E\left(C_{2}-C_{1} \mid C_{1}<C_{2}\right)$ depend on whether an investment was undertaken. Furthermore it is clear that the optimality of such an investment will depend on whether its cost $k$ is higher or lower than the expected gains it promises. Evaluating (9) in the two cases $\delta^{S P A}=1$ and $\delta^{S P A}=1$ yields

$$
B(0)=\frac{(\bar{c}-\underline{c})}{6}
$$

and

$$
B(1)=\frac{7(\bar{c}-\underline{c})}{12}-k,
$$

respectively. Investing will be optimal whenever $B(1)>B(0)$. Consequently, the optimal investment decision is:

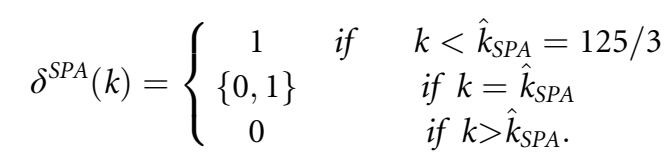

\section{First-price auction}

Analogously, the optimal investment decision in the first-price auction is obtained by looking at the expected profits in the auction, which are given by

$$
B\left(\delta^{F P A}\right)=\Pi_{1} E\left(b_{1}\left(C_{1}\right)-C_{1} \mid C_{1}<C_{2}\right)
$$

Here is the advantaged bidder's bid-function as given by $(3)$ in the case where $\delta^{S P A}=1$ and by (2) in the case where $\delta^{S P A}=0$. It follows in an analogous way to the above that the optimal investment rule is given by

$$
\delta^{F P A}(k)=\left\{\begin{array}{cl}
1 & \text { if } \quad k<\hat{k}_{F P A}=34 \\
\{0,1\} & \text { if } k=\hat{k}_{F P A} \\
0 & \text { if } k>\hat{k}_{F P A} .
\end{array}\right.
$$

22 Obviously it is without loss of generality to assume that player 1 has the right to invest. 


\section{Appendix B: Instructions (FPA treatment)}

Welcome to the experiment! This is an experiment to study behavior of people making decisions. We are only interested in observing how people act on average, not how you act personally. So do not think that we expect you to behave in any particular way. Be aware that your behavior will affect the amount of money you win in this experiment. Thus, it is profitable for you to act in the best way possible. On the following pages you find the instructions on how this experiment works and how to use the computer during the experiment. The instructions are the same for all participants in the experiment.

Please, do not disturb the other participants during the experiment. If you need any help, please raise your hand and wait silently. We will attend you as soon as possible.

How to win money. At the beginning of the experiment, you receive 1000 ptas just for participation. At the end of the experiment you are paid the amount of money you have won during the experiment in addition to these 1000 ptas.

The game

You play 44 rounds of the simple game we explain in the following. In every round you play against another PLAYER from this room. This PLAYER will change in every round. Neither you know whether you have already interacted with nor does the other PLAYER know whether he has already interacted with you. This means that your decisions are anonymous at all times.

At the beginning of each round you receive an initial endowment that can be different in different rounds. Every round of the game consists of two phases, an investment phase and a procurement auction phase. Money you can only win in the procurement auction. The investment phase only enables one of the two PLAYERS to achieve an advantage in the procurement auction. The rounds (remember: every round consists of two phases) are completely independent and nothing you do in one particular round will influence any of the other rounds.

In the investment phase only one of the two players has the opportunity to make an investment at a certain expenditure. The investment will lead the player that has invested to have an advantageous distribution of costs (we explain later on in detail what that 
means). You have the opportunity to invest every two rounds. In the other rounds the other PLAYER has the opportunity to invest.

In the procurement auction, you and the other PLAYER compete for the right to undertake a project. You have to bid in order to determine the winner of the procurement auction and the price to be paid to the winner for undertaking the project. The PLAYER who places the lower bid wins. Realization of the project is costly. This cost will change in every round. It is chosen according to a distribution. Your distribution is the initial distribution if you did not invest (or if did not have the opportunity to invest) and it is the advantageous distribution if you did invest.

If you win the procurement auction, your profit will be the price that is paid to undertake the project less the cost of undertaking it. The investment phase. In the investment phase only one of the two PLAYERS (either the other PLAYER or you) has the opportunity to invest. If the PLAYER who can invest does so, he has the advantageous cost distribution in the procurement auction, while the other one has the initial distribution. If the PLAYER who has the opportunity to invest decides not to do so, both players have the initial distribution.

Now we explain in more detail what it means to have an advantageous distribution.

If the PLAYER who has the possibility to invest decided not to do so, both PLAYERS have the initial distribution. This means that each PLAYER has cost between 300 and 400 ptas, where all numbers in this interval are equally likely.

If the PLAYER who has the opportunity to invest did so, this PLAYER has the advantageous distribution. This means that the PLAYER has cost between 200 and 400 ptas, where again all numbers in this interval are equally likely.

Note that whenever you invest you have a higher probability to have a low cost, which may lead to a higher probability to make more profits in the competition.

In any case the PLAYER who could not invest has the initial distribution, i.e. cost between 300 and 400 ptas.

Investment is costly. The investment expenditure is different in every round and will only be known to the PLAYER who can 
undertake the investment. This is why in every round the PLAYER has to decide again whether the investment seems profitable to him or not. If he decides to invest, the investment expenditure is deducted from the initial endowment he has received at the beginning of the round.

In every round, before the procurement auction, both PLAYERS will be informed about whether the investment has been undertaken or not.

The procurement auction. In the second phase of each round both PLAYERS participate in a procurement auction for an imaginary project. In every round, the cost of undertaking the project is determined randomly for each of the PLAYERS according to his distribution (advantageous or initial). If the PLAYER has the initial distribution his cost is between 300 and 400 ptas. Each number in this interval is equally likely. If he has the advantageous distribution his cost is between 200 and 400 ptas. Again, each number in this interval has the same probability to appear.

Before you decide about your bid, the cost you have in this round appears on your screen. It indicates the amount of money you have to spend to undertake the project (in case you win). The other PLAYER will not be able to observe your cost, nor will you be able to observe his cost. You and the other PLAYER have to decide on your bids, knowing only your own cost and the distribution of cost of the other PLAYER. Who gets the project, and how much money you win depends on your bids as follows.

The procurement auction is always won by the PLAYER who offers to undertake the project at the lower cost, i. e. the player that places the lower bid. The winner is paid his bid. Consequently one can interpret the bid of a player as the amount of money for which he would be willing to undertake the project. Remember that undertaking the project is costly (you observed this cost before placing your bid). The PLAYER who wins the procurement auction has to pay this cost upon undertaking the project. This means that the winner only makes a positive profit from the procurement auction if the price he receives is higher than his cost.

The losing player receives no payment in the procurement auction and incurs no cost. 
What does it mean to have an "advantageous or initial distribution"? We would like you to focus once more on what it means to have an advantageous or initial distribution of cost in the procurement auction. Having the initial distribution means that any number between 300 and 400 ptas has the same probability to appear as your cost in this round. With the advantageous distribution, every number between 200 and 400 ptas will have the same probability to appear.

\begin{tabular}{llcc}
\hline & & $\begin{array}{c}\text { advantageous } \\
\text { distribution }\end{array}$ & $\begin{array}{l}\text { initial } \\
\text { distribution }\end{array}$ \\
\hline The probability & 400 ptas & $100 \%$ & $100 \%$ \\
to have cost & 350 ptas & $75 \%$ & $50 \%$ \\
lower than & 300 ptas & $50 \%$ & $0 \%$ \\
& 250 ptas & $25 \%$ & $0 \%$ \\
\hline
\end{tabular}

As you can see in the table the probability to have cost of less then 350 ptas is $50 \%$ with the initial distribution. While with the advantageous distribution the probability to have a cost of less than 350 ptas is higher, namely $75 \%$.

Note that if you have the advantageous distribution there is a probability of $50 \%$ to have a cost of less then 300 ptas. In this case your cost will be lower than the other player's cost for sure (he has the initial distribution and thus, a cost between 300 and 400 ptas).

Observe that having the advantageous distribution does not necessarily imply that you have a lower cost than the other PLAYER. You will only have a higher probability than with the initial distribution that this is the case. Having a lower cost allows the PLAYER to place a lower bid and thus, have a higher probability of winning. Still the identity of the winner and the profits depends on BOTH bids. $^{23}$

23 The instructions were followed by a number of control questions, and, thereafter, a short summary of the rules. 


\section{Appendix C: Estimation results}

Table 11A.1. Random effects linear regression for treatments FPA and SPA (see equation (7))

\begin{tabular}{lcccc}
\hline & \multicolumn{2}{c}{ FPA } & \multicolumn{2}{c}{ SPA } \\
\cline { 2 - 5 } Bid & firm 1 & firm 2 & firm 1 & firm 2 \\
\hline & $126.66^{* * * *}$ & $139.68^{* * *}$ & $80.63^{* *}$ & $88.65^{* * *}$ \\
$a_{0}$ & $(16.66)$ & $(12.43)$ & $(33.95)$ & $(35.35)$ \\
& $0.67^{* * *}$ & $0.64^{* * *}$ & $0.68^{* * *}$ & $0.69^{*}$ \\
$\beta_{0}$ & $(0.05)$ & $(0.04)$ & $(0.10)$ & $(0.10)$ \\
& $135.14^{* * *}$ & $-32.84^{* *}$ & 21.56 & -12.62 \\
$a_{1}$ & $(19.57)$ & $(15.04)$ & $(38.01)$ & $(39.69)$ \\
& $-0.46^{* * *}$ & $0.08^{*}$ & -0.13 & 0.01 \\
$\beta_{1}$ & $(0.06)$ & $(0.04)$ & $(0.12)$ & $(0.11)$ \\
& $-103.41^{* * *}$ & - & 35.07 & - \\
$a_{2}$ & $(18.85)$ & - & $(30.70)$ & - \\
& $0.37^{* * *}$ & - & -0.04 & - \\
$\beta_{2}$ & $(0.06)$ & - & $(0.10)$ & - \\
$\sigma_{\mathrm{u}}$ & 7.35 & 7.28 & 28.02 & 31.67 \\
$\sigma_{\mathrm{e}}$ & 23.99 & 18.39 & 41.28 & 43.46 \\
$\rho$ & 0.09 & 0.14 & 0.32 & 0.35 \\
$R^{2}$ within & 0.54 & 0.55 & 0.50 & 0.19 \\
$R^{2}$ between & 0.58 & 0.11 & 0.27 & 0.0 \\
$R^{2}$ overall & 0.54 & 0.51 & 0.42 & 0.13 \\
& & & &
\end{tabular}

Table 11A.2. Comparison of treatments with endogenous investment (END) and exogenous asymmetry

\begin{tabular}{lccccc}
\hline & \multicolumn{2}{c}{ FPA } & \multicolumn{2}{c}{ SPA } \\
\cline { 2 - 3 } \cline { 5 - 5 } Bid & firm 1 & firm 2 & firm 1 & firm 2 \\
\hline \multirow{2}{*}{ Cons. } & $245.70^{* * *}$ & $86.51^{* * *}$ & $72.10^{* *}$ & $48.28^{* *}$ \\
& $(12.72)$ & $(9.95)$ & $(24.90)$ & $(24.26)$ \\
Cost & $0.25^{* * *}$ & $0.78^{* * *}$ & $0.70^{* * *}$ & $0.81^{* * *}$ \\
& $(0.05)$ & $(0.03)$ & $(0.09)$ & $(0.07)$ \\
Break & $-111.17^{* * *}$ & - & -64.97 & - \\
& $(22.47)$ & - & $(42.97)$ & - \\
& $0.39^{* * *}$ & - & 0.21 & -
\end{tabular}


Table 11A.2. (cont.)

\begin{tabular}{|c|c|c|c|c|}
\hline \multirow[b]{2}{*}{ Bid } & \multicolumn{2}{|c|}{ FPA } & \multicolumn{2}{|c|}{ SPA } \\
\hline & firm 1 & firm 2 & firm 1 & firm 2 \\
\hline \multirow[t]{2}{*}{ Break*c } & $(0.07)$ & - & $(0.14)$ & - \\
\hline & 13.72 & $21.70 *$ & 30.33 & 26.09 \\
\hline \multirow[t]{2}{*}{ END } & $(17.40)$ & $(13.01)$ & $(31.51)$ & $(30.75)$ \\
\hline & -0.03 & -0.06 & -0.15 & -0.11 \\
\hline \multirow[t]{2}{*}{$E N D * c$} & $(0.07)$ & $(0.04)$ & $(0.12)$ & $(0.09)$ \\
\hline & 26.67 & - & $130.05 * *$ & - \\
\hline \multirow[t]{2}{*}{ Break END } & $(24.60)$ & - & $(44.88)$ & - \\
\hline & -0.07 & - & $-0.40 * *$ & - \\
\hline Break ${ }^{*} E N D{ }^{*} c$ & $(0.07)$ & - & $(0.13)$ & - \\
\hline$\sigma_{u}$ & 12.56 & 7.28 & 29.56 & 27.15 \\
\hline$\sigma_{e}$ & 24.60 & 18.39 & 43.95 & 43.80 \\
\hline$\rho$ & 0.21 & 0.14 & 0.31 & 0.28 \\
\hline$R^{2}$ within & 0.57 & 0.55 & 0.49 & 0.20 \\
\hline$R^{2}$ between & 0.27 & 0.11 & 0.02 & 0.06 \\
\hline$R^{2}$ overall & 0.50 & 0.51 & 0.38 & 0.17 \\
\hline
\end{tabular}

Table 11A.3. Comparison of treatments FPA (SPA) and COMPFPA (COMPSPA)

\begin{tabular}{|c|c|c|c|c|}
\hline \multirow[b]{2}{*}{ Bid } & \multicolumn{2}{|c|}{ FPA } & \multicolumn{2}{|c|}{ SPA } \\
\hline & firm 1 & firm 2 & firm 1 & firm 2 \\
\hline \multirow[t]{2}{*}{ Cons. } & $\begin{array}{l}259.46 * * * \\
(12.32)\end{array}$ & $\begin{array}{c}108.32 * * * * \\
(9.36)\end{array}$ & $\begin{array}{l}101.79: * * * \\
(18.47)\end{array}$ & $\begin{array}{l}73.65^{* * * *} \\
(18.74)\end{array}$ \\
\hline & $0.22 * * *$ & $0.72 * * *$ & $0.55 * * *$ & $0.70 * \cdots$ \\
\hline \multirow[t]{2}{*}{ Cost } & $(0.05)$ & $(0.03)$ & $(0.07)$ & $(0.05)$ \\
\hline & $-101.43 * * *$ & - & 36.45 & - \\
\hline \multirow[t]{2}{*}{ Break } & $(20.47)$ & - & $(30.87)$ & - \\
\hline & $0.36 * * *$ & - & -0.04 & - \\
\hline \multirow[t]{2}{*}{ Break*c } & $(0.07)$ & - & $(0.10)$ & - \\
\hline & 34.88 & -24.48 & -17.02 & -62.38 \\
\hline \multirow[t]{2}{*}{ СОMP } & (25.93) & $(21.43)$ & $(43.61)$ & $(42.04)$ \\
\hline & -0.10 & 0.07 & 0.03 & 0.18 \\
\hline \multirow[t]{2}{*}{$C O M P^{*} c$} & $(0.10)$ & $(0.06)$ & $(0.17)$ & $(0.12)$ \\
\hline & -11.77 & - & $-179.68 * *$ & - \\
\hline
\end{tabular}


Table 11A.3. (cont.)

\begin{tabular}{lccccc}
\hline & \multicolumn{2}{c}{ FPA } & & \multicolumn{2}{c}{ SPA } \\
\cline { 2 - 3 } \cline { 5 - 6 } Bid & firm 1 & firm 2 & & firm 1 & firm 2 \\
\hline Break ${ }^{*}$ COMP & $(41.95)$ & - & & $(59.74)$ & - \\
Break $^{*}$ COMP*c & 0.04 & - & & 0.49 & - \\
$\sigma_{u}$ & $7.11)$ & - & & $(0.17)^{* * *}$ & - \\
$\sigma_{e}$ & 25.81 & 6.92 & & 34.51 & 37.57 \\
$\rho$ & 0.09 & 0.22 & & 41.17 & 42.47 \\
$R^{2}$ within & 0.51 & 0.52 & & 0.41 & 0.44 \\
$R^{2}$ between & 0.41 & 0.32 & & 0.04 & 0.21 \\
$R^{2}$ overall & 0.49 & 0.50 & 0.34 & 0.12 \\
& & & & & 0.12 \\
\hline
\end{tabular}

\section{References}

Arozamena, L. and E. Cantillon (2004), Investment Incentives in Procurement Auctions, Review of Economic Studies 71, 1-18.

Bag, P. (1997). Optimal Auction Design and R \& D, European Economic Review 41, 1655-1674.

Cox, J. C., R. M. Isaac, P.-A. Cech and D. Conn (1996), Moral Hazard and Adverse Selection in Procurement Contracting, Games and Economic Behavior, 17, 147-176.

De Silva, D., T. Dunne and G. Kosmopolou (2003), An Empirical Analysis of Entrant and Incumbent Bidding in Road Constructions Auctions, Journal of Industrial Economics 3, 295-316.

Fischbacher, U. (2007), Z-tree Zurich Toolbox for Readymade Experiments, Experimental Economics 10(2), 171-178.

Grimm, V. (2006), Sequential versus Bundle Auction for Recurring Procurement, University of Cologne Working Paper.

Güth, W., R. Ivanova-Stenzel, and E. Wolfstetter (2005), Bidding behavior in asymmetric auctions: An experimental study, European Economic Review 49, 1891-1913.

Kagel, J.H. (1995), Auctions: A Survey of Experimental Research, in J. Kagel and A. E. Roth (eds.) Handbook of Experimental Economics, Princeton, NJ.

Maskin, E. and J. Riley (2000). Asymmetric Auctions, Review of Economic Studies 67, 439-454. 
Myerson, R. (1981), Optimal Auction Design, Mathematics of Operations Research 6, 58-73.

Piccone, M. and G. Tan (1996). Cost-Reducing Investment, Optimal Procurement and Implementation by Auctions, International Economic Review 37, 663-685.

Tan, G. (1992), Entry and R\&D in Procurement Contracting, Journal of Economic Theory, 58, 41-60. 